\title{
A rare case of giant urethral calculus and multiple urethral diverticulum
}

\author{
A. Agarwal ${ }^{1}$, G. Sigdel ${ }^{1}$, W.K. Belokar ${ }^{2}$ \\ ${ }^{1}$ Mch Resident ${ }^{2}$ Professor and HOD, Department of Urology, College of Medical Sciences, Bharatpur, \\ Nepal
}

\begin{abstract}
Urethral stones in adults are rare and usually encountered with urethral stricture or diverticulum. We report a 54 years old gentleman who presented with urinary retention due to a large urethral calculus impacted in bulbar urethra with multiple stones in anterior and posterior urethral diverticulum. On examination a mass of size $5.5 \mathrm{~cm} \times 4 \mathrm{~cm} \times 3 \mathrm{~cm}$ was palpable at anterior perineum with a fistulous tract from which pus was oozing out. On retrograde urethrogram a large urethral calculus with bulbar diverticulum and multiple radio opacity in prostatic area were revealed. Patient was managed by suprapubic cystostomy initially and later on by external urethrotomy, diverticulectomy, urethroscopic removal of multiple stones in prostatic urethral diverticulum and urethroplasty.
\end{abstract}

Key words: giant urethral calculus, perineal urethrotomy, retrograde urethrogram, urethral diverticulum.

\section{Introduction}

Urethral stones are commonly associated with urinary tract calculi and underlying diverticulum or stricture urethra. ${ }^{1}$ Urethral calculi represent less than $1 \%$ of all urinary stone diseases. Giant urethral calculi are extremely rare. ${ }^{2}$ The majority of urethral calculi occur in males and rarely in females. ${ }^{3}$ We are reporting an interesting case of a giant urethral calculus impacted in bulbar urethra with multiple urethral diverticulum.

\section{Case report}

A 54 year-old gentleman presented in emergency with acute retention of urine. He had history of dysuria, Correspondence: A. Agarwal

E-mail:draaman@gmail.com frequency and two episodes of near urinary retention in past 2 years. There was no history of any operation in perineum and genitalia. On physical examination a hard dull aching mass of size $5.5 \mathrm{~cm} \times 4 \mathrm{~cm} \times 3 \mathrm{~cm}$ was palpable at anterior perineum. Also there was a fistulous tract from which pus was oozing out (fig-1). Urinary bladder was grossly distended. General examination did not reveal any abnormality. Suprapubic stab cystostomy was done in emergency room to relief his discomfort and antibiotics started.

Routine blood investigations and renal function tests were normal. Metabolic work up and serum 
A. Agarwal et al. A rare case of giant urethral calculus and multiple urethral diverticulum

parathyroid hormone and serum calcium were within normal range. Urine culture did not show any growth of organism. Plain CT scan showed a large calculus in the bulbar urethra measuring $5 \times 2.6 \mathrm{~cm}$ and multiple small calculus measuring between $1.5 \times 1.2 \mathrm{~cm}$. Retrograde urethrogram revealed large urethral calculus, bulbar urethral diverticulum with multiple impacted stones and multiple radio-opacity in the region of prostaties urethra (Fig-2). USG abdomen showed bilateral mild hydroureteronephrosis with prostatic calcification.

Under spinal anesthesia perineal urethrolithotomy, diverticulectomy followed by urethroscopy from proximal urethrotomy opening was done. On urethroscopy of prostatic urethra, diverticulums along with multiple stones were found (fig-3). All stones impacted in diverticulum were removed mechanically by using semi-rigid ureteroscope. No attempt was made to excise prostatic urethral diverticulum. The largest urethral stone measured $5.5 \mathrm{cmx} 4 \mathrm{~cm} \times 3 \mathrm{~cm}$ which weighted 36grams (fig-3). Urethra was tapered and repaired in two layers over Foley catheter which was removed after 4 weeks. Biochemical analysis revealed struvite calculus. Patient made rapid and full recovery without any complication.

Figure1: Perineal examination showing giant calculus, suprapubic cystostomy and fistula tract with pus.



Figure 2: Plain CT scan and retrograde urethrogram showing giant urethral calculus, anterior urethral diverticulum with impacted stones and multiple radio-opacity in prostatic urethra

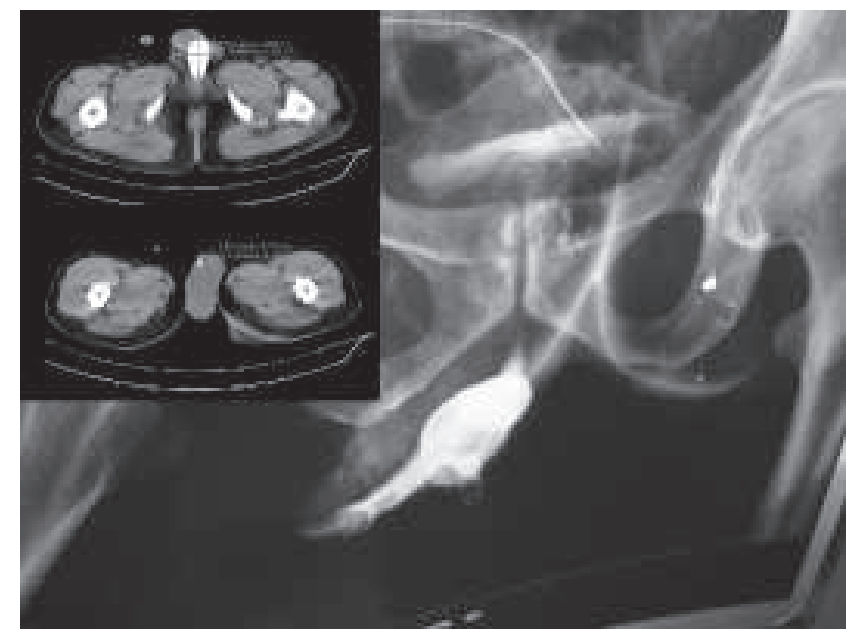

Figure 3: Perineal urethrotomy, stone in anterior urethral diverticulum, urethroscopic view showing stones in prostatic urethral diverticulum and specimen of removed stones from anterior and posterior urethral diverticulum

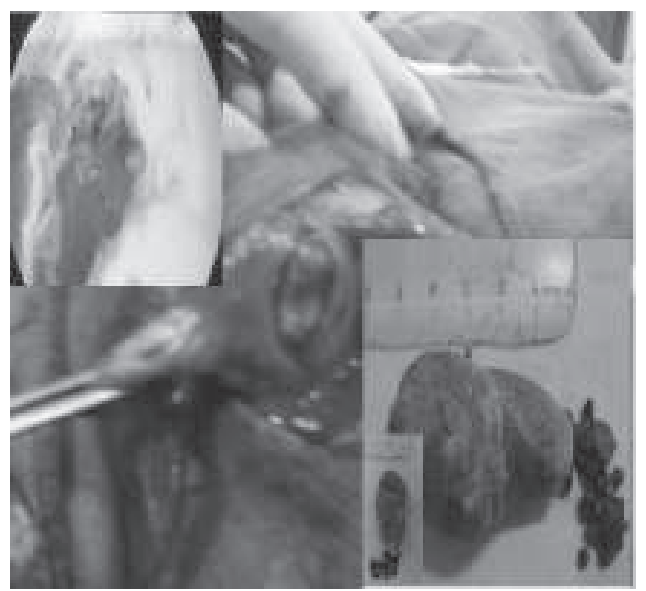

\section{Discussion}

Urethral stones are rare form of urolithiasis accounting for less than $1 \%$ of urinary calculi, but have greater prevalence in developing countries. ${ }^{4}$ Urethral stones in general affect children more often than adults, due to the higher prevalence of bladder stones in this age 
Journal of College of Medical Sciences-Nepal, 2012, Vol-8, No-2

group. ${ }^{5}$ Predisposing factors for in situ development of urethral stones include the presence of urethral diverticulum, urethral stricture, hypospadias and meatal stenosis. ${ }^{6,7}$ They are exceedingly rare in females because of low incidence of vesicle calculi and shorter urethra. ${ }^{8}$ In our case diverticulum seems to be the most probable cause as stricture, hypospadias and meatal stenosis were not found. The possibility of stone migration from the upper urinary tract was considered unlikely as the patient denied any history of ureteric colic and was found to have no renal stones.

Depending upon the site of origin, urethral stones are classified as primary and secondary or migrating. ${ }^{2}$ Primary calculi are associated with urethral abnormalities such as stricture, diverticula, and foreign body. Secondary stones are more common than primary stone and have migrated from upper urinary tract. Urethral calculi are mainly composed of struvite, ${ }^{9}$ calcium phosphate, or calcium carbonate. Primary stones do not cause acute symptoms, while migrant stones may present as acute retention, dysuria, dribbling, or sometimes sepsis in the presence of infection. ${ }^{2}$ There is a report of giant posterior urethral calculus in literature measuring $72 \times 49 \times 52 \mathrm{~mm}$ associated with urethral stricture. ${ }^{10}$

Management of urethral calculi varies according to the site, size and associated urethral disease.

Retrograde manipulation into the urinary bladder followed by litholapaxy or lithotripsy is a suitable procedure for small urethral calculi. Anterior urethral calculi can be removed with instillation of $2 \%$ lignocaine jelly, ventral meatotomy or urethroscopic method. Giant urethral calculi should be treated with open surgery. In urethral stones associated with stricture urethra, stone removal and urethroplasty are preferable. ${ }^{6}$ The treatment of choice for an impacted, large calculus in the bulbar urethra is perineal urethrotomy with urethroplasty as we did in our case. Follow up of 6 months showed no evidence of urethral stricture or recurrent stone in our patient. What makes our case even more interesting is the presence of prostatic urethral diverticulum with impacted stones in it, presence of which could be made out only after urethroscopy. Both retrograde urethrogram and USG abdomen were unable to report this diverticulum as both commented it to be prostatic calcification.

\section{References}

1. Q.J.Ahmed, M.Akhtar, Gulfam, et al. Giant urethral diverticulum with calculi. PakJ Surg 2003;19:106-8.

2. T.V.Shanmugam, V.Dhanapal, T.Rajaraman, et al. Giant urethral calculi. Hospital Medicine 2000; 61:582.

3. H.A.de Carvalho. Giant urethral calculus: a case report $J$ Urol.1977;118:334-5.

4. S.Koga, Y.Arakaki, M.Matsuoka, et al. Urethral calculi. Br J Urol 1990; 65:288-92.

5. B.A.Kamal, R.M.Anikwe, H.Darawani, et al. Urethral calculi: presentation and management. Br J Urol Int 2004; 93: 549-52.

6. A. Hegele, P.Olbert, S.Wille, et al. Giant calculus of the posterior urethra following recurrent penile urethral stricture. Urol Int 2002; 69:160-1.

7. G.L.Larkin, J.E.Weber. Giant urethral calculus. A rare case of urinary retention.J Emeg Med 1996;14:707-9.

8. T.Win. Giant urethral calculus: Singapore Med J 1994; 35:414-5.

9. M.F.Usta, M.Baykara, T.Erdogru, et al. Idopathic primary giant calculi in young male patient. Int Urol Nephrol 2005;37:295-7.

10. A. Bolukbasi, B.Gumus. Giant posterior urethral calculus. BJU1990;65:301-2. 